\title{
Significance of miR-148a in Colorectal Neoplasia: Downregulation of miR-148a Contributes to the Carcinogenesis and Cell Invasion of Colorectal Cancer
}

\author{
Yumi Hibino $^{\text {a }}$ Naoya Sakamoto $^{\text {a }}$ Yutaka Naito $^{\text {a }}$ Keisuke Goto ${ }^{a}$ \\ Htoo Zarni Oo ${ }^{a}$ Kazuhiro Sentani ${ }^{a}$ Takao Hinoi $^{b}$ Hideki Ohdan $^{b}$ \\ Naohide Oue ${ }^{a}$ Wataru Yasui ${ }^{a}$ \\ Departments of a Molecular Pathology and ${ }^{\mathrm{b}}$ Gastroenterological and Transplant Surgery, Applied Life Sciences, \\ Institute of Biomedical and Health Sciences, Hiroshima University, Hiroshima, Japan
}

\section{Key Words}

MicroRNAs $\cdot$ Colorectal cancer $\cdot$ miR-148a $\cdot$ Matrix

metalloproteinase 7

\begin{abstract}
Objective: Colorectal cancer (CRC) develops through the deregulation of gene expression and the accumulation of epigenetic abnormalities, leading to tumor cell acquisition of malignant features. MicroRNAs (miRNAs) play a critical role in cancer development, where they can act as oncogenes or oncosuppressors. Methods: miR-148a expression was measured by qRT-PCR in patients with colorectal adenoma $(\mathrm{n}=$ 21) and CRC (stage I-IV, $n=159$ ) using formalin-fixed paraffin-embedded tissue samples. In situ hybridization (ISH) using an miR-148a-specific probe was also performed. To further confirm the direct effect of miR-148a on matrix metalloproteinase (MMP)7 expression in CRC, MTT and cell invasion assays using HT29 and WiDr cells were performed. Results: miR-148a expression was found to be clearly downregulated in high-grade adenoma compared to low-grade adenoma on both qRT-PCR and ISH analysis. Downregulation of miR-148a expression was significantly correlated with
\end{abstract}

(C) 2015 S. Karger AG, Basel

$1015-2008 / 15 / 0825-0233 \$ 39.50 / 0$ advanced clinicopathological features and was an independent prognostic classifier in patients with stage III CRC. In CRC cells and tissues, miR-148a expression was inversely correlated with the expression of MMP7. Conclusion: We showed the collaborative participation of miR-148a and MMP7 in CRC cell invasion. These results also demonstrate that the downregulation of miR-148a expression promotes CRC progression, especially carcinogenesis and cancer cell invasion.

(c) 2015 S. Karger AG, Basel

\section{Introduction}

Colorectal cancer (CRC) is a leading cause of cancer mortality and the third-most common cancer worldwide with $>900,000$ cases occurring annually, nearly 500,000 of which are fatal. It is assumed that CRC originates from a sequential accumulation of molecular and genetic alterations to colon epithelial cells [1]. A deeper understanding of the pathogenesis and biological features of CRC is necessary to further inform and enhance early detection and treatment methods.

\section{KARGER 125}

E-Mail karger@karger.com www.karger.com/pat 
MicroRNAs (miRNAs) are involved in the pathogenesis and progression of multiple types of cancers [2]. Growing evidence suggests that miRNAs have a novel role as oncogenes or tumor suppressors [3]. The pattern of miRNA expression can be useful to classify various types of cancers, and these profiles can have prognostic and therapeutic implications. Compared with mRNA, a modest number of miRNAs may be sufficient for clinical purposes. Interestingly, miRNAs remain largely intact in different tissues and are virtually unaffected by RNA degradation [4]. Formalin-fixed, paraffin-embedded (FFPE) tissue samples have been collected over decades of routine histopathological examinations and are the most widely available tissue samples for use in clinical research; studies performed on paired frozen and FFPE specimens have shown very similar results [5]. This is because the obstacle is likely due to high fragmentation but fragmentation does not cause a further loss of quality when naturally occurring small RNAs are targeted. All of these features make miRNAs a very promising tool for large-scale and clinical cohort/trial-based studies.

In this decade, differential expression of miRNAs has been investigated in CRC $[6,7]$ and it has been reported that some of the deregulated miRNAs are involved in CRC progression $[8,9]$. Among these, we have focused on miR-148a, in particular, because we have reported that it directly targets matrix metalloproteinase (MMP)7 and participates in tumor invasion in gastric cancer [10]. It is well known that the level of MMP7 correlates with advanced clinical stages and poor clinical outcome in CRC $[11,12]$; miR-148a could be involved in CRC progression through the regulation of MMP7 expression. The progression to CRC, however, is considered to be a stepwise process, the so-called adenoma-carcinoma sequence [13, 14]. Although this sequence is characterized by the progressive acquisition of genetic and epigenetic changes, including the deregulation of miRNA, the changes in miRNA expression and their timing are largely unknown. As for miR-148a, several lines of evidence have shown that its expression is downregulated in most types of cancer, including CRC [15-17]. However, the expression of $\mathrm{miR}-148 \mathrm{a}$ in precancerous regions of the colon remains unclear.

In this study, to confirm the relationship between miR-148a and CRC prognosis, we analyzed miR-148a expression in 159 FFPE specimens from CRC cases by qRTPCR and compared them with corresponding nonneoplastic mucosa samples. The associations between miR148a expression and clinicopathological factors, including prognosis, were also investigated. We then verified the
Table 1. Clinicopathological characteristics of 159 CRC cases

\begin{tabular}{|c|c|c|c|c|}
\hline & \multirow{2}{*}{$\begin{array}{l}\text { Cases, } \\
\mathrm{n}(\%)\end{array}$} & \multicolumn{3}{|c|}{ Cases with miR-148a expression, $\mathrm{n}$} \\
\hline & & low & high & $\mathrm{p}$ value ${ }^{2}$ \\
\hline \multicolumn{5}{|l|}{ Age, years } \\
\hline $63(29-89)^{1}$ & & & & 0.4994 \\
\hline$<63$ & & 46 & 26 & \\
\hline$>63$ & & 60 & 27 & \\
\hline \multicolumn{5}{|l|}{ Sex } \\
\hline Male & $97(61)$ & 49 & 13 & 0.0071 \\
\hline Female & $62(39)$ & 57 & 40 & \\
\hline \multicolumn{5}{|l|}{ Location } \\
\hline Right & $31(19)$ & 24 & 7 & 0.1463 \\
\hline Left & $128(81)$ & 82 & 46 & \\
\hline \multicolumn{5}{|l|}{$\mathrm{T}$ grade } \\
\hline $\mathrm{T}_{1}$ & $22(14)$ & $30\left(\mathrm{~T}_{1 / 2}\right)$ & $26\left(\mathrm{~T}_{1 / 2}\right)$ & \\
\hline $\mathrm{T}_{2}$ & $34(21)$ & $76\left(\mathrm{~T}_{3 / 4}\right)$ & $27\left(\mathrm{~T}_{3 / 4}\right)$ & 0.0104 \\
\hline $\mathrm{T}_{3}$ & $74(47)$ & & & \\
\hline $\mathrm{T}_{4}$ & $29(18)$ & & & \\
\hline \multicolumn{5}{|l|}{$\mathrm{N}$ grade } \\
\hline No & $93(58)$ & 54 & 39 & \\
\hline N1 & $8(5)$ & $52(\mathrm{~N} 1 / 2 / 3)$ & $14(\mathrm{~N} 1 / 2 / 3)$ & 0.0067 \\
\hline $\mathrm{N} 2$ & $17(11)$ & & & \\
\hline N3 & $41(26)$ & & & \\
\hline \multicolumn{5}{|l|}{ Stage } \\
\hline I & $47(30)$ & 25 & 22 & \\
\hline II & $42(26)$ & 81 (II-IV) & 31 (II-IV) & 0.0266 \\
\hline III & 49 (31) & & & \\
\hline IV & $21(13)$ & & & \\
\hline
\end{tabular}

${ }^{1}$ Range. ${ }^{2}$ Fisher's exact test.

relationship between miR-148a expression and MMP7, both in the clinical samples and in vitro. Furthermore, we analyzed miR-148a expression in 21 colorectal adenoma samples and showed when such expression is downregulated in colorectal neoplasia.

\section{Materials and Methods}

Tissue Samples

A total of 159 primary tumor and corresponding nonneoplastic mucosa samples were collected from patients diagnosed with CRC who were treated at Hiroshima University Hospital between 1997 and 2003. Tumor staging was according to the TNM classification system. Clinical details of these CRC patients are summarized in table 1. For qRT-PCR, 159 CRC samples, the corresponding normal adjacent samples and 21 colorectal adenoma samples were used. Samples were FFPE tissues from 159 patients who had undergone surgical excision for CRC and from 21 
colorectal adenoma patients who had received endoscopic mucosal resection. Details are described in the online supplementary information (for all online suppl. material, see www.karger.com/ doi/10.1159/000438826).

\section{MiRNA Extraction and $q R T-P C R$}

Tumor areas were macrodissected from $20-\mu \mathrm{m}$-thick FFPE tissue samples with sterile disposable scalpels and then subjected to RNA isolation using a RecoverAll total nucleic acid isolation kit (Ambion, Tex., USA).

Extracted miRNA was reverse-transcripted using a TaqMan microRNA reverse transcription kit (Applied Biosystems, Foster City, Calif., USA) according to the manufacturer's protocol. HsamiR-148a was detected using TaqMan MicroRNA assays. The relative miR-148a expression levels were calculated by the formula $2^{-\Delta \Delta \mathrm{CT}}$, where $\Delta \Delta \mathrm{CT}=\Delta \mathrm{CT}$ miR-148a $-\Delta \mathrm{CT}$ RNU48, and $\mathrm{a} \geq 32$ CT value was interpreted as an amplification too low to quantify. Further details have been described previously [18] and in the online supplementary information.

\section{Immunohistochemistry}

Immunohistochemical analysis was performed with a Dako EnVision $^{\mathrm{TM}}+$ mouse peroxidase detection system (Dako Cytomation, Calif., USA). Sections were incubated with mouse monoclonal anti-human MMP7 antibody (mouse monoclonal: dilution 1:50; Daiichi Fine Chemical, Takaoka, Japan) for $1 \mathrm{~h}$ at room temperature, followed by incubation with EnVision+ anti-mouse peroxidase for $1 \mathrm{~h}$. A result was considered positive if at least $10 \%$ of the cells were stained. Details are described in the online supplementary information.

Immunofluorescence Staining and in situ Hybridization

A biotin $3^{\prime}$-labeled, locked nucleic acid-incorporated miRNA probe (miR-CURY LNA detection probe, Exiqon, Mass., USA) was used for the visualization of miR-148a. A 6-pmol probe was hybridized with the $1 \times$ Enzo in situ hybridization buffer (Exiqon). Immunological detection was performed using the Dako GenPoint $^{\mathrm{TM}}$ tyramide signal amplification system for biotin-labeled probes (Dako, Carpinteria, Calif., USA). For the detection of miR148 a in combination with immunofluorescence, after signal amplification with biotin tyramide, sections were incubated with primary antibodies against CAM5.2 (Becton Dickinson Labware, Franklin Lakes, N.J., USA), followed by incubations with Alexa Fluor 546-conjugated streptavidin and Alexa Fluor 488-conjugated goat anti-mouse IgG (Molecular Probes, Eugene, Oreg., USA). Further details have been described previously [19] and also appear in the online supplementary information.

\section{Cell Lines}

HT29 and WiDr cell lines derived from human CRC were used, and maintained in RPMI-1640 (Nissui Pharmaceutical, Tokyo, Japan) containing a $10 \% \mathrm{FBS}$ sphere of $5 \% \mathrm{CO}_{2}$ and $95 \%$ air at $37^{\circ} \mathrm{C}$.

\section{Western Blotting}

HT29 was lysed as described previously [10]. The membrane was incubated with primary anti-MMP7 antibody (dilution 1:500; Daiichi Fine Chemicals). Immunocomplexes were visualized with an ECL Western blot detection system (Amersham Biosciences, Piscataway, N.J., USA). Details are described in the online supplementary information.

Importance of miR-148a in CRC

\section{Cell Transfection}

Transfection of cells was performed with Lipofectamine RNAiMAX reagent (Invitrogen, Carlsbad, Calif., USA), according to the manufacturer's instructions. Pre-miR-148a and negative control miRNA (Ambion) were used for each transfection at a final concentration of $100 \mathrm{nM}$. The cells were seeded at $50 \%$ confluence on the day before transfection. Details are described in the online supplementary information.

\section{Cell Invasion and Growth Assay}

The cells were seeded at a density of 2,000 cells/well in 96 -well plates. Transiently transfected cells were plated at $1 \times 10^{6}$ cells/well in RPMI-1640 medium with no serum in the upper chamber of a transwell insert ( 8 - $\mu \mathrm{m}$ pore diameter; Chemicon, Temecula, Calif., USA) coated with Matrigel. Cell growth was monitored after 1, 2 and 4 days by MTT assay. Details are described in the online supplementary information.

Statistical Methods

Details are described in the online supplementary information. $\mathrm{p}<0.05$ was considered to be statistically significant.

\section{Results}

\section{Comparison of miR-148a Expression Levels in}

Nonneoplastic Colon Mucosa, Colorectal Adenoma and CRC Tissues

To further understand the relationship between miR148a expression and clinicopathological parameters, we analyzed the miR-148a expression in 159 FFPE samples of primary CRC and their corresponding nonneoplastic colon mucosa by qRT-PCR. Significant downregulation of miR-148a expression was detected in 113 of 159 CRC cases $(71.1 \%)$ when compared with the corresponding nonneoplastic mucosa (fig. 1a). We also examined miR148a expression in 21 colorectal adenoma samples and compared it with its expression in CRC and nonneoplastic colon mucosa samples. It was observed that miR-148a expression was clearly downregulated in high-grade adenoma compared to low-grade adenoma (nonneoplastic mucosa vs. colorectal adenoma: $\mathrm{p}<0.05$, nonneoplastic mucosa vs. CRC: $\mathrm{p}<0.001$ and low-grade adenoma vs. high-grade adenoma: $\mathrm{p}<0.001$, respectively; fig. $1 \mathrm{~b}, \mathrm{c}$ ). Then, to confirm the downregulation of miR-148a expression in tissue sections, we performed ISH for miR148a, using sections from nonneoplastic colon mucosa, low-grade adenoma, high-grade adenoma and CRC. Consistent with the qRT-PCR data, we observed clear miR-148a positivity in normal tissues and weak positivity in low-grade adenoma. However, the high-grade adeno$\mathrm{ma}$ and CRC sections were completely negative for miR148a (fig. 2). These data indicate that miR-148a down- 

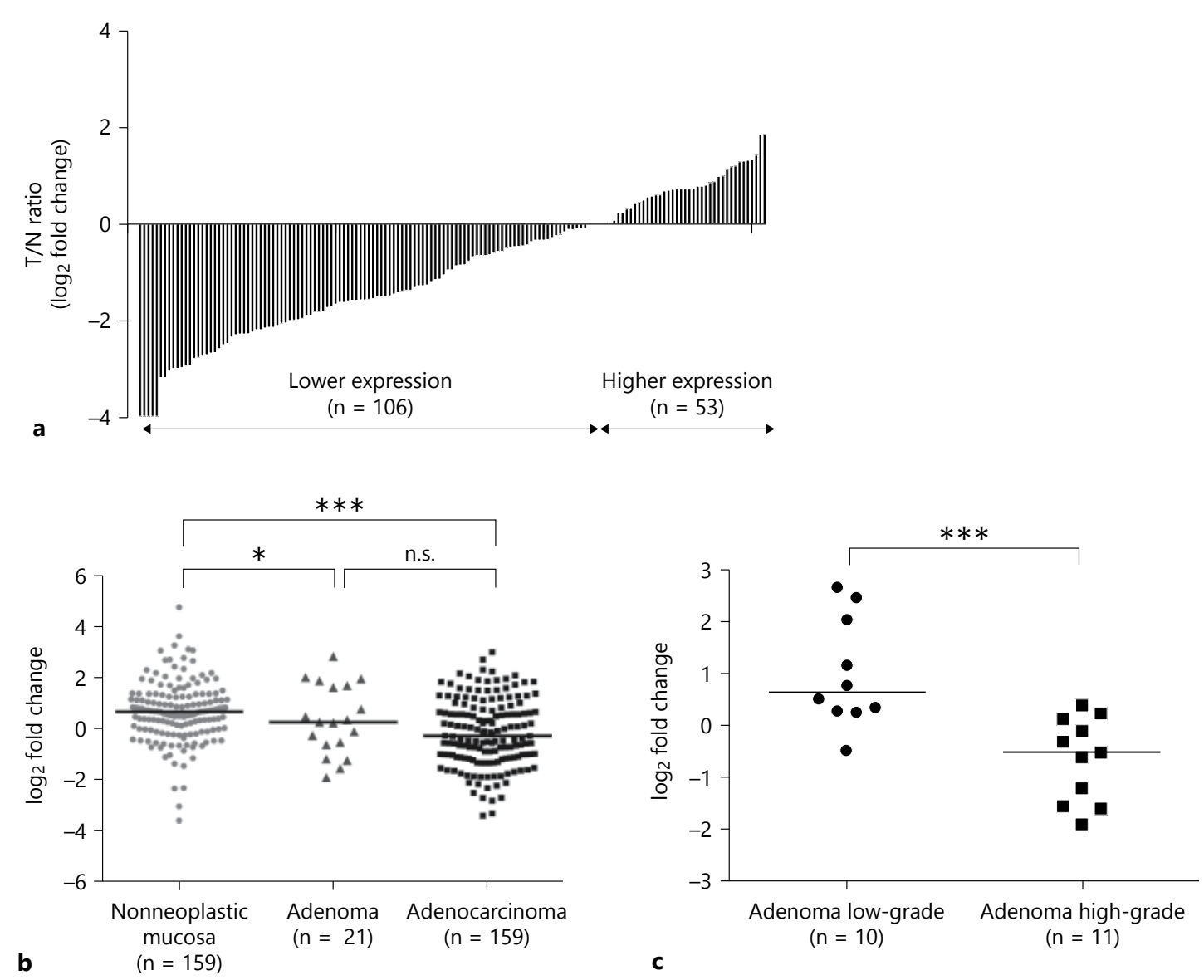

Fig. 1. miR-148a expression in non-neoplastic colon mucosa, colorectal adenoma and CRC tissue. a Comparison between miR148a expression in nonneoplastic colon mucosa and CRC tissues by Western blotting showing the T-to- $\mathrm{N}$ ratio in $159 \mathrm{CRC}$ cases. b Comparison between miR-148a expression in nonneoplastic co-

lon mucosa, colorectal adenoma and CRC tissues. n.s. $=$ Not significant. ${ }^{*} \mathrm{p}<0.05{ }^{* * *} \mathrm{p}<0.001$. c Comparison between miR-148a expression in low- and high-grade colorectal adenoma. ${ }^{* * *} \mathrm{p}<$ 0.001 .

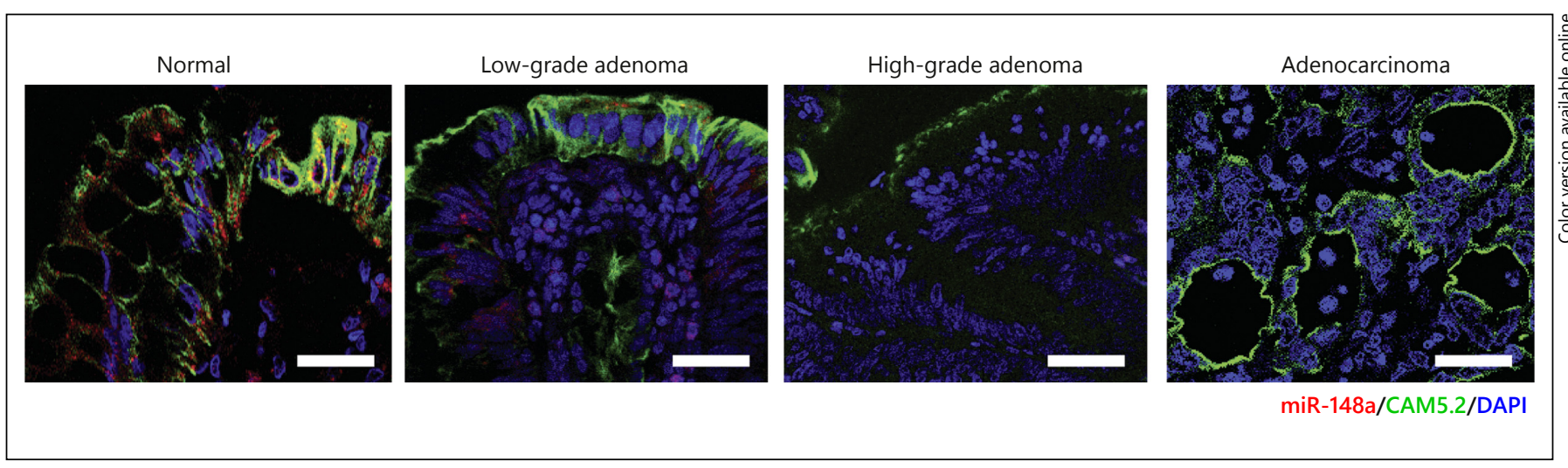

Fig. 2. miR-148a expression in nonneoplastic colon mucosa, colorectal adenoma and CRC tissue detected by ISH in combination with immunofluorescence. Scale bars $50 \mu \mathrm{m}$. Green: CAM5.2, Red: miR-148a, Blue: DAPI. 


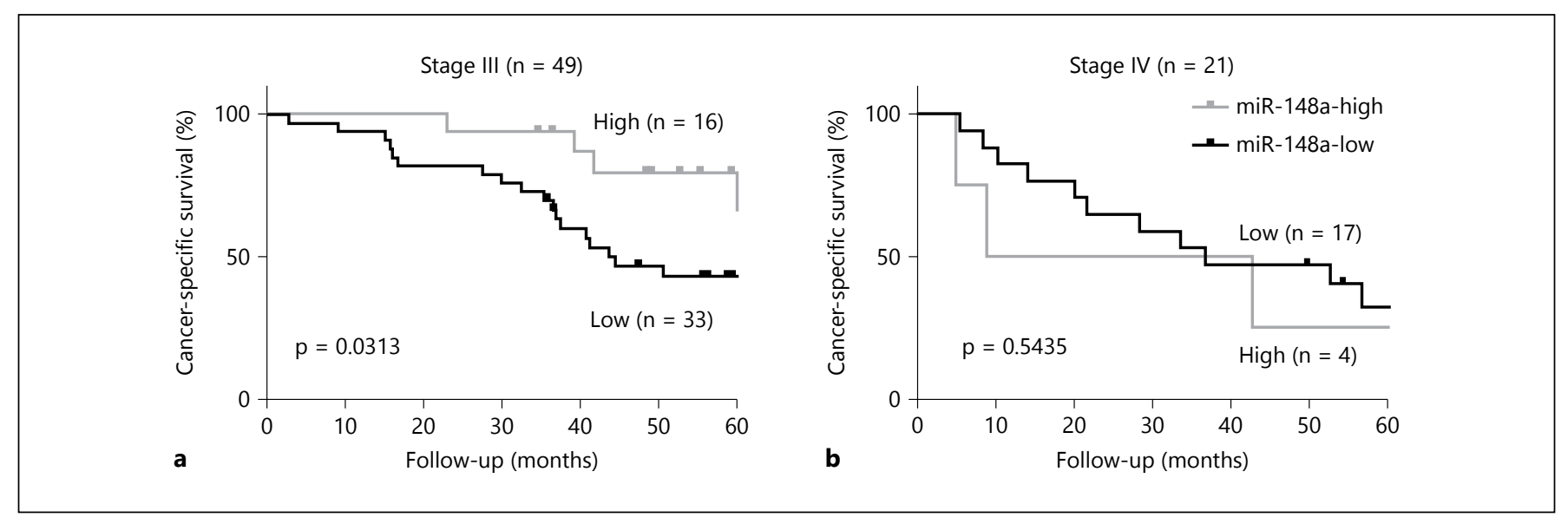

Fig. 3. Relationship between miR-148a expression and CRC prognosis. A log-rank test and Kaplan-Meier plots were constructed for the miR-148a-high and miR-148a-low groups. Cancer-specific survival of 49 patients with stage III CRC (a) and 21 patients with stage IV CRC (b) based on the expression levels of miR-148a (cutoff line $=$ a higher tertile miR-148a expression level). regulation may be involved in the mechanism of the shift from low-grade adenoma to high-grade adenoma, and that it plays an important role in the carcinogenesis of CRC.

\section{Clinicopathological Characteristics of CRC}

Cases in Which miR-148a Expression Levels Are

Downregulated

To further understand the relationship between miR-148a expression and clinicopathological parameters, we analyzed the correlation between miR-148a expression levels and clinicopathological factors in 159 CRC cases. Figure 1a shows that miR-148a was downregulated in approximately two thirds of the cases, so we divided the objective CRC cases into 2 groups (i.e. with low and high expression levels of miR-148a) based on the higher tertile miR-148a expression of the group. The relationship between the miR-148a expression and clinicopathological parameters of CRC are summarized in table 1. MiR-148a low CRC cases were more advanced in terms of $\mathrm{pT}$ grade $(\mathrm{p}=0.0104), \mathrm{pN}$ grade $(\mathrm{p}=$ $0.0067)$ and $\mathrm{pStage}(\mathrm{p}=0.0266)$ than miR-148a high CRC cases.

We also evaluated the association between miR-148a expression and cancer-specific mortality in CRC cases. In all 159 cases and in 112 patients with advanced CRC, there was no significant difference in prognosis between those whose CRC tissue samples exhibited lower miR148a expression levels and those whose samples exhibited higher expression (data not shown). However, when the sampling was limited to the 49 stage III cases, i.e. the cases with lymph node metastasis, we found a significant correlation between prognosis and the difference of miR-148a expression ( $p=0.0313$; fig. 3a). However, when limited to the 21 stage IV samples, i.e. cases with distant metastasis, there was no significant correlation between the 2 factors (fig. $3 \mathrm{~b}$ ). In order to evaluate the potential use of miR-148a expression as a prognostic predictor in patients with stage III CRC, both univariate and multivariate Cox proportional hazards analyses were used to further investigate the association of miR148a expression with cancer-specific mortality (table 2 ). According to the univariate analysis, $\mathrm{T}$ grade and $\mathrm{miR}$ 148a expression were significantly associated with cancer-specific mortality. According to the multivariate model, miR-148a expression was an independent prognostic classifier of cancer-specific mortality. These results indicate that loss of miR-148a may play a critical role in CRC progression.

\section{Correlation between miR-148a and MMP7 in CRC}

To confirm the correlation between miR-148a and MMP7 in CRC, we performed MMP7 immunohistochemistry and ISH with an miR-148a-specific probe in sections of nonneoplastic colon and CRC tissue. On ISH, we were only able to detect miR-148a expression in the nonneoplastic colon epithelium, not in the cancer cells. The staining pattern of MMP7 was completely opposite in the nonneoplastic and the cancer tissue (fig. 4a). It was also found that upregulation of MMP7 was inversely associated with miR-148a expression in the CRC cases analyzed (fig. 1a; table 3). To further confirm the di- 
Table 2. Univariate and multivariate analysis of factors influencing survival in 49 patients with stage III CRC

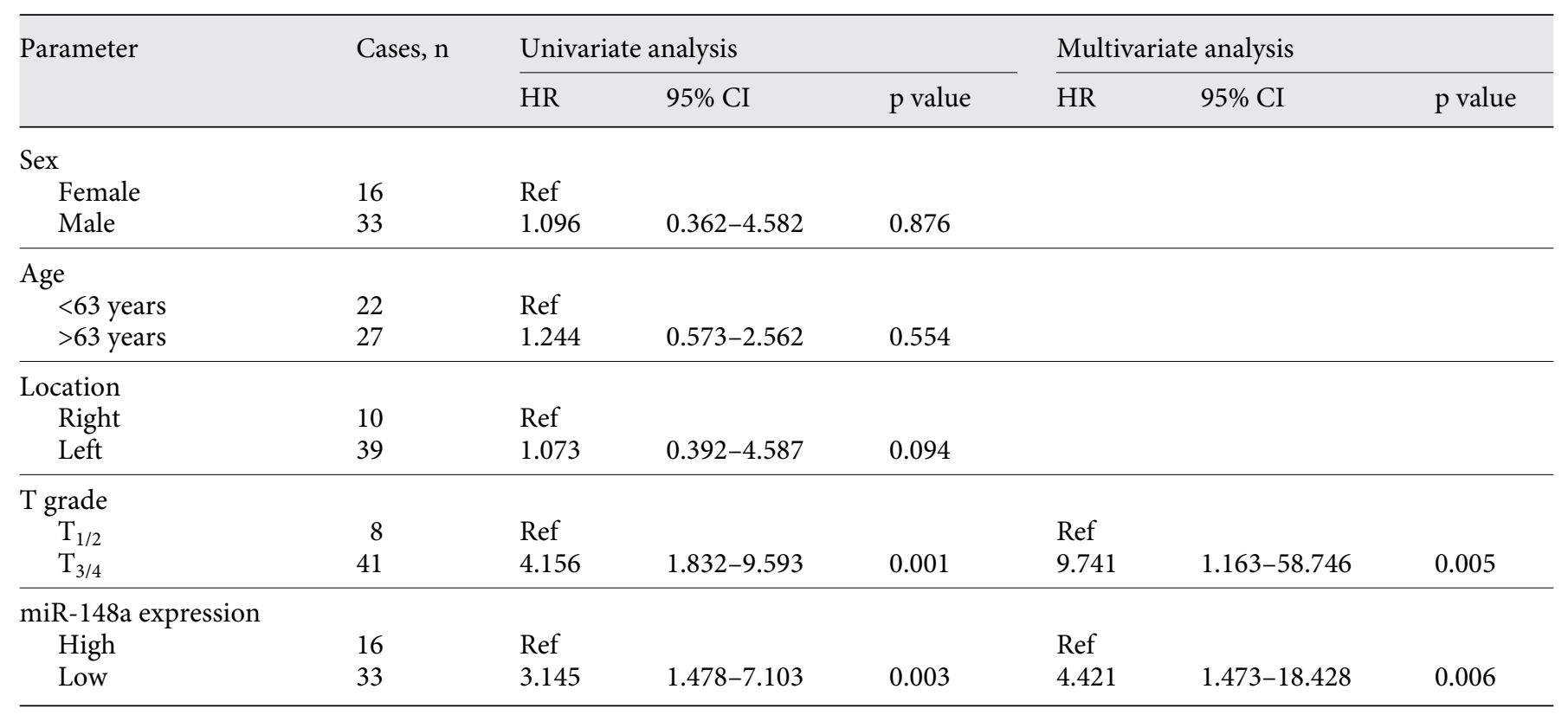

Tumor grade was classified according to the criteria of the International Union against Cancer TNM classification of malignant tumors. $\mathrm{CI}=$ Confidence interval; $\mathrm{HR}=$ hazard ratio.

rect effect of miR-148a on MMP7 expression and the biological significance of MMP7 expression in CRC, we performed MTT and cell invasion assays. MMP7 expression was noted in the HT29 and WiDr cells of 5 CRC cell lines. Three lines of siRNAs (siRNA1-3) were transfected into these cells, and all of them were able to suppress the endogenous MMP7 expression. HT29 and WiDr cells were transfected to induce miR-148a overexpression, then examined for the MMP7 expression level by Western blotting. MMP7 expression was significantly downregulated; levels were similar to those of MMP7specific siRNA-treated cells (online suppl. fig. 1). We used only siRNA1 in the following experiments to knock down the endogenous MMP7 because all 3 siRNA were effective to the same extent. Although cell viability was not significantly different between miR-148a-overexpressed or MMP7 siRNA-transfected and negative-control HT29 cells (data not shown), the invasive ability was markedly repressed by miR-148a overexpression similar to the case of MMP7-specific siRNA-treated cells (HT29: day $1 \mathrm{p}<0.01$ and day $2 \mathrm{p}<0.001$; WiDr: day $1 \mathrm{p}<0.001$ and day $2 \mathrm{p}<0.001$; fig. $4 \mathrm{~b}$ ). These observations support that miR-148a directly regulates MMP7 expression in CRC cells and that these cooperatively contribute to CRC invasion.

\section{Discussion}

We reported here that miR-148a expression was significantly downregulated in CRC tissues and that this was tightly correlated with advanced clinicopathological features and poor clinical outcome in stage III patients. We also showed that downregulation of miR-148a expression was inversely correlated with MMP7 overexpression, and that the timing of this downregulation seemed to correspond with the development of high-grade adenoma. To our knowledge, this is the first report that mentions the transition of miR-148a expression in the precancerous region in the colon. In this study, our data indicate that miR-148a downregulation is an early event in colorectal carcinogenesis. This is supported by the fact that the average miR-148a expression in high-grade adenomas was similar to that in the CRC specimens, and that no miR148a expression could be detected in high-grade adenoma or CRC by ISH. The methylation of miR-148a CpG sites has been proposed as a potential mechanism for its inactivation in CRC [15]. Downstream targets of miR148a have been increasingly understood and characterized. Overexpression of DNMT1 and LSD1 caused by miR-148a downregulation has been reported [20, 21], which could play a critical role in epigenetic machinery 


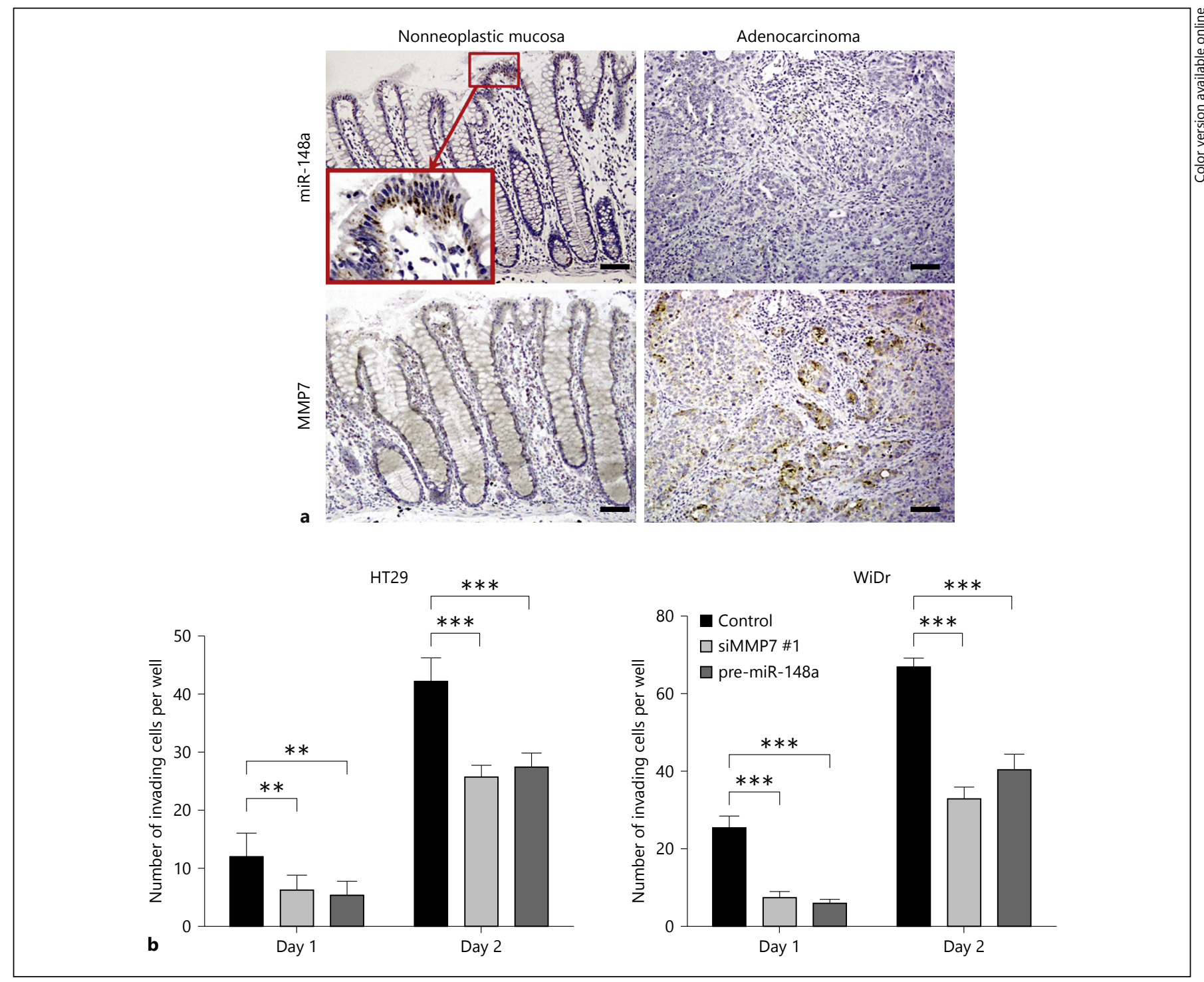

Fig. 4. Expression pattern of miR-148a and MMP7 in nonneoplastic colon mucosa and CRC tissue, and effect of miR-148a on MMP7 expression and CRC cell invasiveness. a ISH using miR-148a-specific probe and immunohistochemistry using anti-MMP7 antibody on the nonneoplastic colon mucosa and CRC tissue. Scale bars
$100 \mu \mathrm{m}$. b Effect of miR-148a overexpression and MMP7 knockdown on cell invasion of HT29 and WiDr cells. HT29 and WiDr transfected with negative control miRNA, anti-miR-148a and MMP7-specific siRNA were incubated in Boyden chambers. Results are mean \pm SD of triplicate measurements. ${ }^{* *} \mathrm{p}<0.01 .^{* * *} \mathrm{p}<0.001$. and be involved in further miR-148a downregulation. Accordingly, it was assumed that this methylation of miR-148a CpG sites contributes to the beginning of CRC carcinogenesis. However, it has also been reported that even patients with stage IV CRC do not always show hypermethylation of its promoter (although miR-148a was most frequently downregulated in patients with stage IV CRC) [15]. While further studies are needed to elucidate a fuller picture of the molecular mechanisms involved in the carcinogenesis of CRC, miR-148a could be a promis-

Importance of miR-148a in CRC
Table 3. Correlation between the expression of MMP7 and miR148a in CRC tissue

\begin{tabular}{clll}
\hline & \multicolumn{2}{l}{ miR-148a } & \\
\cline { 2 - 4 } & low & high & p value $^{1}$ \\
\hline MMP7 & 46 & 34 & 0.0183 \\
Low & 60 & 19 & \\
High & & & \\
\hline
\end{tabular}

${ }^{1}$ Fisher's exact test. 
ing candidate as a key regulator of the carcinogenesis in CRC.

It has been reported that miR-148a downregulation is a statistically significant prognostic factor in both stage III/IV and stage II/III CRC cohorts [15, 22]. In this study, we intended to determine the significance of miR-148a downregulation in CRC cases without any stage limitation, but it was finally found that its downregulation was an independent prognostic factor only in patients with stage III CRC. It has been well established that adjuvant chemotherapy after surgery decreases recurrence and improves survival in patients with stage III CRC [23], and most of the patients with stage III CRC in our cohort actually received this treatment (data not shown). Several studies have reported that miR-148a plays important roles in chemosensitivity to anticancer drugs, such as cisplatin, paclitaxel and 5-FU [24-26]. In addition, it has been suggested that miR-148a could be an effective suppressor of the tyrosine kinase receptor/ AKT signaling pathway through the direct targeting of ERBB3 and IGF1R and the inhibition of both EGF- and IGF-induced activation of AKT [27-29]. There has been a recent and rapid development in biological agents targeted against components of receptor tyrosine kinasesignaling cascades in the treatment of CRC. Currently, only KRAS mutational status is used as a decisive marker for EGFR inhibitor therapy in CRC patients. However, it has subsequently become clear that only $13-17 \%$ of CRC cases without KRAS mutations in codon 12 or 13 of exon 2 responded to EGFR inhibitor therapy [30]. Based on these previous reports, it is presumed that miR-148a plays an important role in drug resistance against a wide range of anticancer drugs including molecular-targeted therapy, and that it could be a predictive marker for the therapeutic benefit of these treatments.

Given the evidence that miR-148a is preferentially silenced in CRC tissues due to promoter methylation, we performed functional studies to explore the potential tumor suppressor features of miR-148a. We discovered that the restoration of miR-148a expression in HT29, a cell line with rather low miR-148a expression and high MMP7 expression, indeed resulted in a significant decrease in cell invasion. Although we mentioned the correlation between miR-148a downregulation and AKT as above, it is also well known that the activation of the AKT/JNK pathway induces MMP7 expression [31,32]. Taken together with the findings of our previous report, this suggests that miR-148a downregulation affects MMP7 overexpression both directly and indirectly; promoting robust MMP7 ex- pression in CRC tissue and supporting the invasiveness of CRC cells.

In summary, we have shown that miR-148a downregulation is an independent prognostic classifier in patients with stage III CRC. Furthermore, this study identified the timing of miR-148a downregulation in the adenoma-carcinoma sequence of CRC. Our findings provide novel evidence for the contribution of miR-148a not only as a key regulator of cell invasion but also a trigger for the carcinogenesis of CRC.

\section{Acknowledgements}

We thank Mr. Shinichi Norimura for excellent technical assistance. This work was carried out with the kind cooperation of the Research Center for Molecular Medicine, Faculty of Medicine, Hiroshima University. We thank the Analysis Center of Life Science, Hiroshima University, for the use of their facilities. This work was supported by Grants-in-Aid for Research from the Ministry of Education, Culture, Science, Sports and Technology of Japan and, in part, by a Grant-in-Aid for the Third Comprehensive 10-Year Strategy for Cancer Control and Cancer Research from the Ministry of Health, Labor and Welfare of Japan and the National Institute of Biomedical Innovation (Program for Promotion of Fundamental Studies in Health Sciences). This work was also supported in part by a Research Fellowship of the Japan Society for the Promotion of Science and the National Cancer Center Research and Development Fund (23-A-9).

\section{Disclosure Statement}

The authors declare no conflict of interest.

References

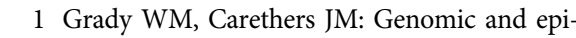
genetic instability in colorectal cancer pathogenesis. Gastroenterology 2008;135:1079-1099.

$\checkmark 2$ Calin GA, Croce CM: MicroRNA signatures in human cancers. Nat Rev Cancer 2006;6: $857-866$.

3 Di Leva G, Croce CM: Roles of small RNAs in tumor formation. Trends Mol Med 2010;16: 257-267.

$\checkmark 4$ Doleshal M, Magotra AA, Choudhury B, Cannon BD, Labourier E, Szafranska AE: Evaluation and validation of total RNA extraction methods for microRNA expression analyses in formalin-fixed, paraffin-embedded tissues. J Mol Diagn 2008;10:203-211.

5 Weng L, Wu X, Gao H, Mu B, Li X, Wang JH Guo C, Jin JM, Chen Z, Covarrubias M, Yuan YC, Weiss LM, Wu H: MicroRNA profiling of clear cell renal cell carcinoma by whole-genome small RNA deep sequencing of paired frozen and formalin-fixed, paraffin-embedded tissue specimens. J Pathol 2010;222:41-51. 
6 Schetter AJ LS, Sohn JJ, Zanetti KA, Bowman ED, Yanaihara N, Yuen ST, Chan TL, Kwong DL, Au GK, Liu CG, Calin GA, Croce CM, Harris CC: MicroRNA expression profiles associated with prognosis and therapeutic outcome in colon adenocarcinoma. JAMA 2008; 299:425-436.

7 Bartley AN, Yao H, Barkoh BA, Ivan C, 17 Mishra BM, Rashid A, Calin GA, Luthra R, Hamilton SR: Complex patterns of altered microRNA expression during the adenomaadenocarcinoma sequence for microsatellitestable colorectal cancer. Clin Cancer Res 2011;17:7283-7293.

8 Oue N, Anami K, Schetter AJ, Moehler M, Okayama H, Khan MA, Bowman ED, Mueller A, Schad A, Shimomura M, Hinoi T, Aoyagi K, Sasaki H, Okajima M, Ohdan H, Galle PR, Yasui W, Harris CC: High miR-21 expression from FFPE tissues is associated with poor survival and response to adjuvant chemotherapy in colon cancer. Int J Cancer 2014;134:19261934.

-9 Liu X, Zhang Z, Sun L, Chai N, Tang S, Jin J, $\mathrm{Hu} \mathrm{H}$, Nie Y, Wang X, Wu K, Jin H, Fan D: MicroRNA-499-5p promotes cellular invasion and tumor metastasis in colorectal cancer by targeting FOXO4 and PDCD4. Carcinogenesis 2011;32:1798-1805.

10 Sakamoto N, Naito Y, Oue N, Sentani K, Uraoka N, Zarni Oo H, Yanagihara K, Aoyagi K, Sasaki H, Yasui W: MicroRNA-148a is downregulated in gastric cancer, targets MMP7, and indicates tumor invasiveness and poor prognosis. Cancer Sci 2014; 105:236-243.

-11 Martinez-Fernandez A, Garcia-Albeniz X, Pineda E, Visa L, Gallego R, Codony-Servat J, Auge JM, Longaron R, Gascon P, Lacy A, Castells A, Maurel J: Serum matrilysin levels predict outcome in curatively resected colorectal cancer patients. Ann Surg Oncol 2009;16: 1412-1420.

12 Huang Y, Yu H, Lei H, Xie C, Zhong Y: Matrix metalloproteinase 7 is a useful marker for 5-fluorouracil-based adjuvant chemotherapy in stage II and stage III colorectal cancer patients. Med Oncol 2014;31:824.

$\checkmark 13$ Fearon ER, Vogelstein B: A genetic model for colorectal tumorigenesis. Cell 1990;61:759767.

14 Brenner H, Kloor M, Pox CP: Colorectal cancer. Lancet 2014;383:1490-1502.

-15 Takahashi M, Cuatrecasas M, Balaguer F, Hur K, Toiyama Y, Castells A, Boland CR, Goel A: The clinical significance of MiR-148a as a predictive biomarker in patients with advanced colorectal cancer. PLoS One 2012;7:e46684.
16 Liffers ST, Munding JB, Vogt M, Kuhlmann JD, Verdoodt B, Nambiar S, Maghnouj A, Mirmohammadsadegh A, Hahn SA, Tannapfel A: MicroRNA-148a is down-regulated in human pancreatic ductal adenocarcinomas and regulates cell survival by targeting CDC25B. Lab Invest 2011;91:1472-1479.

17 Heo MJ KY, Koo JH, Yang YM, An J, Lee SK, Lee SJ, Kim KM, Park JW, Kim SG: microRNA-148a dysregulation discriminates poor prognosis of hepatocellular carcinoma in association with USP 4 overexpression. Oncotarget 2014;5:2792-2806.

18 Shinmei S, Sakamoto N, Goto K, Sentani K, Anami K, Hayashi T, Teishima J, Matsubara A, Oue N, Kitadai Y, Yasui W: MicroRNA-155 is a predictive marker for survival in patients with clear cell renal cell carcinoma. Int J Urol 2013;20:468-477.

19 Naito Y, Sakamoto N, Oue N, Yashiro M, Sentani K, Yanagihara K, Hirakawa K, Yasui W: MicroRNA-143 regulates collagen type III expression in stromal fibroblasts of scirrhous type gastric cancer. Cancer Sci 2014;105:228235.

20 Yan J, Guo X, Xia J, Shan T, Gu C, Liang Z, Zhao W, Jin S: MiR-148a regulates MEG3 in gastric cancer by targeting DNA methyltransferase 1. Med Oncol 2014;31:879.

21 Balaguer F, Link A, Lozano JJ, Cuatrecasas M, Nagasaka T, Boland CR, Goel A: Epigenetic silencing of miR-137 is an early event in colorectal carcinogenesis. Cancer Res 2010; 70:6609-6618.

22 Tsai HL, Yang IP, Huang CW, Ma CJ, Kuo $\mathrm{CH}$, Lu CY, Juo SH, Wang JY: Clinical significance of microRNA-148a in patients with early relapse of stage II stage and III colorectal cancer after curative resection. Transl Res 2013;162:258-268.

23 Moertel CG FT, Macdonald JS, Haller DG, Laurie JA, Goodman PJ, Ungerleider JS, Emerson WA, Tormey DC, Glick JH, Veeder $\mathrm{MH}$, Mailliard JA: Levamisole and fluorouracil for adjuvant therapy of resected colon carcinoma. N Engl J Med 1990;322:352-358.

24 Fujita Y, Kojima K, Ohhashi R, Hamada N, Nozawa Y, Kitamoto A, Sato A, Kondo S, Kojima T, Deguchi T, Ito M: MiR-148a attenuates paclitaxel resistance of hormone-refractory, drug-resistant prostate cancer PC3 cells by regulating MSK1 expression. J Biol Chem 2010;285:19076-19084.

25 Hummel R, Watson DI, Smith C, Kist J, Michael MZ, Haier J, Hussey DJ: miR-148a improves response to chemotherapy in sensitive and resistant oesophageal adenocarcinoma and squamous cell carcinoma cells. J Gastrointest Surg 2011;15:429-438.
26 Langer C, Radmacher MD, Ruppert AS, Whitman SP, Paschka P, Mrozek K, Baldus CD, Vukosavljevic T, Liu CG, Ross ME, Powell BL, de la Chapelle A, Kolitz JE, Larson RA, Marcucci G, Bloomfield CD: High BAALC expression associates with other molecular prognostic markers, poor outcome, and a distinct gene-expression signature in cytogenetically normal patients younger than 60 years with acute myeloid leukemia: a Cancer and Leukemia Group B (CALGB) study. Blood 2008;111:5371-5379.

27 Xu Q, Jiang Y, Yin Y, Li Q, He J, Jing Y, Qi YT, Xu Q, Li W, Lu B, Peiper SS, Jiang BH, Liu LZ: A regulatory circuit of miR-148a/152 and DNMT1 in modulating cell transformation and tumor angiogenesis through IGF-IR and IRS1. J Mol Cell Biol 2013;5:3-13.

$28 \mathrm{Yu}$ J LQ, Xu Q, Liu L, Jiang B: miR-148a inhibits angiogenesis by targeting ERBB3. J Biomed Res 2011;25:170-177.

29 Zhang JP, Zeng C, Xu L, Gong J, Fang JH, Zhuang SM: MicroRNA-148a suppresses the epithelial-mesenchymal transition and metastasis of hepatoma cells by targeting Met/ Snail signaling. Oncogene 2014;33:40694076.

30 De Roock W CB, Bernasconi D, De Schutter J, Biesmans B, Fountzilas G, Kalogeras KT, Kotoula V, Papamichael D, Laurent-Puig P, Penault-Llorca F, Rougier P, Vincenzi B, Santini D, Tonini G, Cappuzzo F, Frattini M, Molinari F, Saletti P, De Dosso S, Martini M, Bardelli A, Siena S, Sartore-Bianchi A, Tabernero J, Macarulla T, Di Fiore F, Gangloff AO, Ciardiello F, Pfeiffer P, Qvortrup C, Hansen TP, Van Cutsem E, Piessevaux H, Lambrechts D, Delorenzi M, Tejpar S: Effects of KRAS, BRAF, NRAS, and PIK3CA mutations on the efficacy of cetuximab plus chemotherapy in chemotherapy-refractory metastatic colorectal cancer: a retrospective consortium analysis. Lancet Oncol 2010;11: 753-762.

31 Chang MC, Chen CA, Chen PJ, Chiang YC, Chen YL, Mao TL, Lin HW, Lin Chiang WH, Cheng WF: Mesothelin enhances invasion of ovarian cancer by inducing MMP-7 through MAPK/ERK and JNK pathways. Biochem J 2012;442:293-302.

32 Lin MC, Wang FY, Kuo YH, Tang FY: Cancer chemopreventive effects of lycopene: suppression of MMP-7 expression and cell invasion in human colon cancer cells. J Agric Food Chem 2011;59:11304-11318. 